\title{
Cardiovascular dysfunction and liver transplantation
}

\author{
Hye-Mee Kwon and Gyu-Sam Hwang \\ Department of Anesthesiology and Pain Medicine, Laboratory for Cardiovascular Dynamics, Asan Medical Center, \\ University of Ulsan College of Medicine, Seoul, Korea
}

Cardiovascular complications have emerged as the leading cause of death after liver transplantation, particularly among those with advanced liver cirrhosis. Therefore, a thorough and accurate cardiovascular evaluation with clear comprehension of cirrhotic cardiomyopathy is recommended for optimal anesthetic management. However, cirrhotic patients manifest cardiac dysfunction concomitant with pronounced systemic hemodynamic changes, characterized by hyperdynamic circulation such as increased cardiac output, high heart rate, and decreased systemic vascular resistance. These unique features mask significant manifestations of cardiac dysfunction at rest, which makes it difficult to accurately evaluate cardiovascular status. In this review, we have summarized the current knowledge of heart and liver interactions, focusing on the usefulness and limitations of cardiac evaluation tools for identifying high-risk patients.

Keywords: Autonomic nervous system; Cardiovascular dysfunction; Coronary artery disease; Echocardiography; Liver transplantation; Ventriculo-arterial coupling.

\section{Importance of Cardiovascular Function in Liver Transplant Candidates}

Liver transplantation (LT) is the only life-saving treatment option in patients with advanced liver disease. Deceased-donor LT is not frequent but is increasing in Asian countries. Because current liver allocation policies follow the severity principle wherein patients at highest risk for mortality receive top priority, anesthesiologists may face severely ill patients more frequently with deceased-donor LT than with living-donor LT. In

Corresponding author: Gyu-Sam Hwang, M.D., Ph.D.

Department of Anesthesiology and Pain Medicine, Laboratory for Cardiovascular Dynamics, Asan Medical Center, University of Ulsan College of Medicine, 88, Olympic-ro 43-gil, Songpa-gu, Seoul 05505, Korea

Tel: 82-2-3010-3868, Fax: 82-2-3010-6790

Email: kshwang@amc.seoul.kr

ORCID: https://orcid.org/0000-0002-3627-1107

Received: August 8, 2017.

Revised: September 22, 2017.

Accepted: October 12, 2017.

Korean J Anesthesiol 2018 April 71(2): 85-91

https://doi.org/10.4097/kjae.2018.71.2.85 this regard, with the outstanding surgical success of recent LT, cardiovascular complications have emerged as the leading cause of death after LT, particularly among those with advanced liver cirrhosis $[1,2]$. A large national cohort study of the Organ Procurement and Transplantation Network database also revealed that death from cardiovascular disease is the leading cause of early mortality (40\%), followed by infection (28\%), and graft failure (12\%) [3]. Moreover, the numbers of patients receiving LT for non-alcoholic fatty liver disease [4] and alcoholic liver disease are increasing worldwide. Specifically, these patients are associated with one or more cardiovascular risk factors such as diabetes mellitus, hypertension, prior cardiovascular disease, left ventricular hypertrophy, age $>60$ years, smoking, hypertension, and dyslipidemia $[4,5]$.

\section{Understanding Cirrhotic Cardiomyopathy}

As liver cirrhosis (LC) progresses, cardiac dysfunction concomitant with pronounced systemic hemodynamic changes, characterized by hyperdynamic circulation such as increased cardiac output, high heart rate (HR), and decreased systemic vascular resistance (SVR) is well known to appear. These unique features may mask significant manifestations of cardiac

(c) This is an open-access article distributed under the terms of the Creative Commons Attribution Non-Commercial License (http://creativecommons.org/ licenses/by-nc/4.0/), which permits unrestricted non-commercial use, distribution, and reproduction in any medium, provided the original work is properly cited. 
dysfunction because decreased SVR reduces afterload burden to myocardial contraction at rest. However, when challenged by physical stress, such as exercise or surgery, a blunted cardiac contractile response may show limited ability to increase ventricular contractility [6-8]. This phenomenon is known as cirrhotic cardiomyopathy, which encompasses systolic dysfunction diastolic dysfunction, and electromechanical abnormalities, which are typically identified based on a reduced ejection fraction (EF) [9], an E/A ratio < 1 [10], and electrocardiographic changes such as a prolonged QTc interval [11]. Diagnostic and supportive criteria for cirrhotic cardiomyopathy proposed by the 2005 World Congress of Gastroenterology are shown in Table 1 [12].

As cardiovascular complications are leading causes of nongraft related mortality after LT [2], the importance of identifying masked intrinsic cardiac dysfunction or decreased capacity of cardiac contractility due to external stress has been emphasized. However, detecting ventricular dysfunction in a resting state is difficult, as marked vasodilation and increased arterial compliance lead to latent or mild cardiac manifestations [13]. Different stress tests, using drugs or exercise, have been applied to unmask cardiac dysfunction; however, achieving a target HR and blood pressure is difficult given the poor functional conditions of patients with LC [14].

Dobutamine stress echocardiography (DSE) is recommended to discriminate high-risk patients with ischemic heart disease; however, the accuracy of DSE varies widely among studies as a result of various selection criteria [12] and the inability to achieve the predicted target HR to provoke wall motion abnormalities. This inadequacy is based on the failure of beta receptors to respond to sympathetic stimulation in patients with LC or the use of beta blockers to prevent variceal bleeding. Therefore, the accuracy of DSE is questionable, and its sensitivity is reported as low as $13-14 \%[15,16]$. Nicolau-Raducu et al. [17] demonstrated that DSE has $9 \%$ sensitivity, $33 \%$ positive predictive value, and $89 \%$ negative predictive value for predicting early cardiac events after LT.
Nuclear myocardial perfusion imaging with the injection of vasodilators, such as adenosine or dipyridamole, has controversial value for further vasodilation with adenosine; thus, it may be inadequate in already-vasodilated patients with LC $[18,19]$.

\section{QT Interval and LT}

QT interval prolongation is a characteristic manifestation of cirrhotic cardiomyopathy. Since Day et al. [20] first reported the association between prolonged QT interval and alcoholic cirrhosis, QT interval prolongation has been found in about half of all cirrhotic patients, regardless of etiology and is associated with the severity of liver disease [21]. Numerous factors, such as increased sympathetic activity, electrolyte abnormalities, bile salts, hyperinsulinemia, and endotoxin, are involved in the pathogenesis of prolonged QT interval [21-23]. Patients with QT interval prolongation are at greater risk for malignant ventricular arrhythmias and sudden cardiac death and have poorer survival among the cirrhotic population [21].

The QT interval progressively increases from the pre-anhepatic stage and remains prolonged in each stage of LT compared to baseline during LT surgery. In the anhepatic stage, $54 \%$ of recipients show marked prolongation of QTc $\geq 500 \mathrm{~ms}$, which may increase the potential for developing severe ventricular dysrhythmias [24]. Given that arrhythmogenic potential develops rapidly at graft reperfusion just before the start of hypotension during LT [25], special attention is required to prevent severe arrhythmias in patients with a prolonged QT interval. In this regard, the clinical impact of severe QT prolongation $(\geq 500 \mathrm{~ms})$ in the cirrhotic population is an area of further research interest and remains to be established.

Another index of myocardial repolarization lability, known as the QT interval variability index (QTVI), represents the relationship between the QT interval and HR variability [26]. QTVI is a marker of temporal inhomogeneity in patients with ventricular repolarization and an abnormality associated with re-entrant malignant ventricular arrhythmia. Huh et al. [27]

Table 1. Diagnostic and Supportive Criteria for Cirrhotic Cardiomyopathy, Proposed by the 2005 World Congress of Gastroenterology

\begin{tabular}{ll}
\hline \multicolumn{1}{c}{ Diagnostic criteria } & \multicolumn{1}{c}{ Supportive criteria } \\
\hline Systolic dysfunction & Electrophysiological abnormalities \\
Resting ejection fraction $<55 \%$ & Abnormal response to chronotropy \\
Inadequate increase in cardiac output in response to exercise, & Electromechanical uncoupling and/or dyssynchrony \\
volume challenge, or pharmacological stimuli & QTc interval prolongation \\
Diastolic dysfunction & Structural deterioration \\
Early diastolic/atrial filling ratio $<1.0$ (adjusted by age) & Left atrium enlargement and/or left ventricular hypertrophy \\
Prolonged deceleration time $(>200 \mathrm{~ms})$ & Increased myocardial mass \\
Prolonged isovolumetric relaxation time $(>80 \mathrm{~ms})$ & Increased brain natriuretic peptide and/or troponin I
\end{tabular}

Recommended definition of cirrhotic cardiomyopathy: cardiac dysfunction in patients with cirrhosis, in the absence of other known cardiac disease, characterized by blunted contractile responsiveness to stress and/or impaired diastolic relaxation with electrophysiological abnormalities. 
demonstrated a parallel relationship between QTVI and liver cirrhosis severity, which may indicate a link between the severity of liver cirrhosis and increased vulnerability to arrhythmias.

\section{Pretransplant Echocardiography and LT}

Although measuring left ventricular EF is the gold standard for assessing systolic function via echocardiography, it is an afterload-dependent index and thus might be insensitive to detect systolic dysfunction in the resting state of patients with LC, because it is normal or elevated in patients with advanced LC $[13,28,29]$. In this regard, a recent intriguing study reported that an elevated EF is associated with worse outcomes. Bushyhead et al. [30] reported that increased EF is associated with a numerical but nonsignificant increased risk of development of stage 4 or 5 chronic kidney disease (sub-hazard ratio, 1.11 per $5 \%$ increase in LVEF; 95\% CI, 0.99-1.24; $\mathrm{P}=0.07$ ). In addition, $\mathrm{EF} \geq 65 \%$ is the best cutoff for increased risk for chronic kidney disease (sub-hazard ratio, 1.75; 95\% CI, 1.06-2.89; P = 0.03). They also demonstrated that greater than a mild degree of tricuspid regurgitation is associated with significantly increased post-transplant mortality in multivariate Cox regression models, and increasing pulmonary artery systolic pressure is associated with a significantly increased risk for hospitalization for myocardial infarction or heart failure.

Speckle-tracking echocardiographic techniques, such as those based on peak systolic longitudinal strain, have been advocated to identify cardiac dysfunction in patients with LC at the resting state, which is considered to more accurately reflect systolic function than conventional methods [31]. Jansen et al. [32] documented a positive correlation between left ventricle contractility, evaluated by speckle tracking echocardiography, and Child-Pugh score severity. In that study, patients with increased myocardial contractility showed reduced transplant-free survival, which suggests that increased contractility might be a tool to identify pre-transplant patients at higher risk. The authors speculated that increased contractility in the cirrhotic population is a sign of a decompensated cardiac function with sympathetic hyperactivation. Therefore, another reliable test to discriminate cardiac dysfunction is required in this specific population.

Diastolic dysfunction is a characteristic pattern of cirrhotic cardiomyopathy. The underlying mechanism of diastolic dysfunction in patients with LC is considered to be increased myocardial wall stiffness attributable to myocardial hypertrophy, fibrosis, and subendothelial edema resulting in high filling pressures of the left ventricle and atrium [10]. Dowsley et al. [33] reported that elevated markers of diastolic dysfunction during a pre-LT echocardiographic evaluation are associated with excess risk for heart failure and post-LT mortality; early mitral inflow velocity/mitral annular velocity (E/e' ratio) $>10$ and a left atrial volume index $\geq 40 \mathrm{ml} / \mathrm{m}^{2}$ are associated with a 3.4-fold and 2.9fold increased risk for developing heart failure after LT, respectively.

\section{Ventriculo-arterial Coupling in LT Candidates}

The left ventricle and systemic arteries are anatomically connected in the cardiovascular system; therefore, their interactions are key determinants of cardiovascular homeostasis in a normal human heart $[34,35]$. The concept of "ventriculo-arterial coupling (VAC)," which integrates not only ventricular performance but also effective arterial load and, most importantly, their interplay, is demonstrated in patients with LC [29]. Shin et al. [29] provided new insight into assessing cirrhotic cardiomyopathy to discriminate worse outcomes from a conventional echocardiographic examination in the resting state, without using preoperative stress tests such as DSE or nuclear myocardial perfusion imaging with adenosine.

When analyzing the pressure-volume curve relationship, determining an exact slope of end-systolic pressure-volume relation (ESPVR) is startlingly too invasive, requiring two invasive catheters in the left ventricle and inferior vena cava to make an occlusive balloon in the human heart. Therefore, invasive ESPVR is thought to be reasonable only in animal studies or during open heart surgery. In this regard, Shin et al. [29] employed a noninvasive technique based on the pressure-volume curve relationship of the single-beat methodology proposed by Chen et al. [36].

Ventricular elastance (Ees), calculated as the ratio of endsystolic pressure (ESP) to end-systolic volume, is an index of load insensitive ventricular contractility [35], and arterial elastance (Ea), the ratio of ESP to stroke volume, is an index of the net measure of arterial load [37]. ESP is defined as $0.9 \times$ systolic blood pressure determined by a noninvasive brachial blood pressure measurement. Therefore, VAC (the ratio of Ees to Ea) could be measured simply by conventional echocardiography [36].

Shin et al. [29] observed that cirrhotic patients have significantly lower Ees, Ea, and VAC values than those of matched-control patients with normal liver function. In patients with a high model for end-stage liver disease (MELD) score (> 25), those with a VAC $>0.61$ had poorer survival outcomes than patients with a VAC $<0.50$, and this was independently associated with the risk of mortality. It is important to understand that neither Ees nor Ea alone but the interaction of the coupling $(=\mathrm{Ea} / \mathrm{Ees})$ value was a significant prognosticator of post-LT non-survivors in that study [29]. Specifically, an increase in afterload (Ea) without an increase in contractility (Ees) leads to ventricular-arterial uncoupling (higher VAC) with a significant reduction in stroke volume, ultimately resulting in increased 
overall mortality during long-term follow-up, particularly in patients with high MELD scores.

In LC patients, afterload (Ea) frequently increases without an elevation in contractility (Ees) when the sympathetic nervous system is progressively activated, with increased circulating concentrations of norepinephrine as LC severity increases, or use of norepinephrine/terlipressin in patients with hepatorenal syndrome. Such an increase in afterload (Ea) burdens the cirrhotic heart and may unmask ventricular dysfunction.

The VAC study by Shin et al. [29] highlights that cirrhotic cardiomyopathy is often latent but can be manifested in situa-

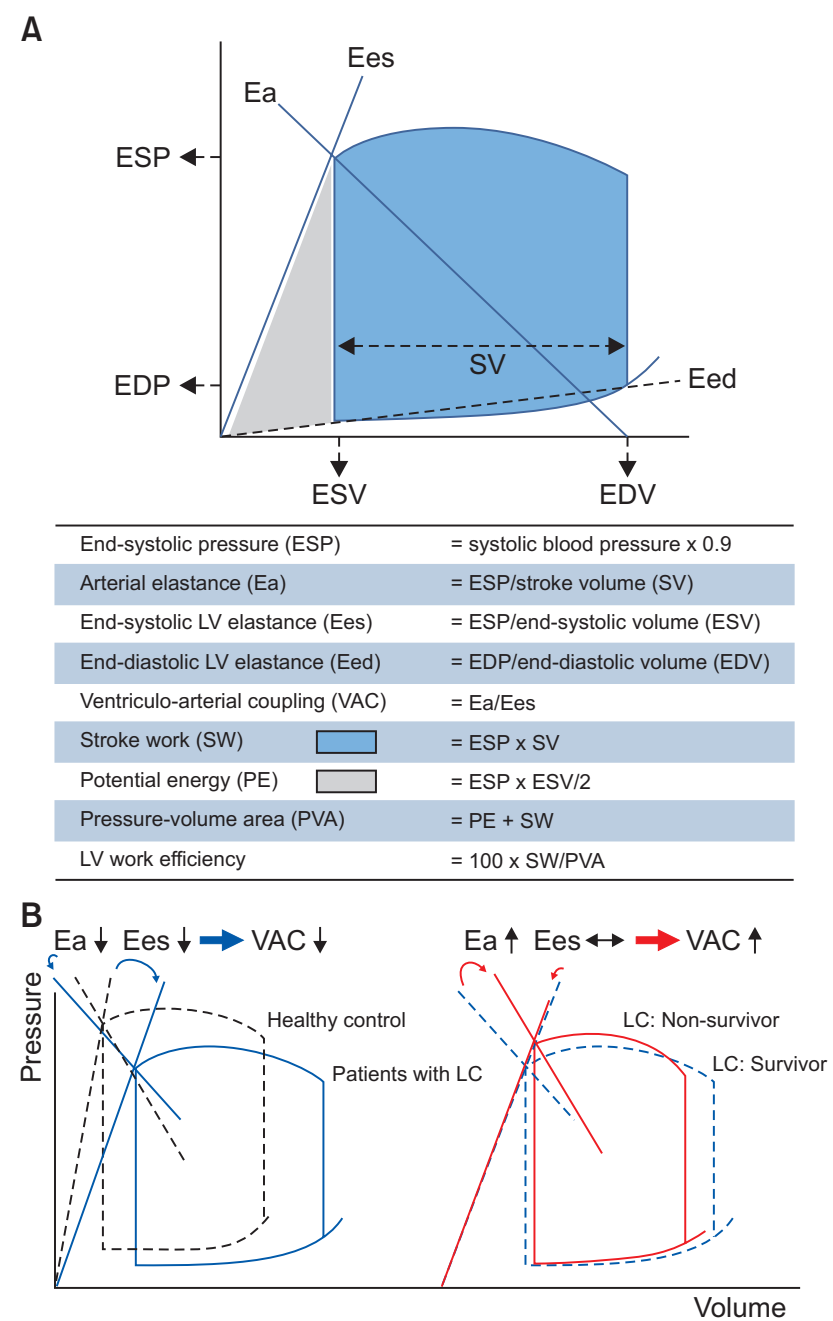

Fig. 1. Pressure-volume loops of the left ventricle (LV). (A) Measurements of parameters derived from a pressure-volume loop of the LV. (B) Example of pressure-volume loops. Compared to a healthy control (black dashed lines), patients with liver cirrhosis (LC) have decreased Ees, $\mathrm{Ea}$, and ventriculo-arterial coupling (VAC) on a right-shifted pressurevolume loop (blue lines). Compared to survivors, Ees is similar but Ea is higher in patients who died (red lines). Consequently, VAC increases and stroke volume decreases compared to values for survivors (blue dashed lines). EDP: end diastolic pressure. Figure from Shin et al. [29] with permission. tions with increased afterload (Fig. 1). Integrating both ventricular and arterial components when assessing cirrhotic cardiomyopathy should be considered important, and its simplicity and non-invasiveness without additional stress loading to unmask cardiac dysfunction further highlights its importance [29,36].

Similarly, Yotti et al. [38] demonstrated that an increase in afterload after phenylephrine injection results in a decrease in systolic function and cardiac output. They applied the new noninvasive measures of the left ventricular systolic function using the difference in echocardiographic ejection intraventricular pressure and validated this procedure using an invasive pressure-volume catheterization study.

\section{Autonomic Nervous System and HR in Patients with LC}

The autonomic nervous system is an important regulator of cardiovascular homeostasis, and an HR analysis is considered a surrogate of vagal and sympathetic disturbances. Therefore, HR measurements have been recognized as a prognostic factor in many clinical investigations [39-42]. Studies showing reduced HR variability, which correlates with disease severity, central hypovolemia, and the degree of portal hypertension have also been reported $[43,44]$. Kim et al. [45] found that sympathetic withdrawal is associated with hypotension after graft reperfusion during LT.

Arterial baroreflex function is also an important contributor to short-term cardiovascular and blood pressure stability. Patients with alcoholic cirrhosis are associated with reduced baroreflex sensitivity, which is significantly related to hemodynamics and biochemical characteristics [46]. Song et al. [47] demonstrated that changes in cardiovagal baroreflex sensitivity are related to increased ventricular mass in patients with LC. In their study, baroreflex sensitivity was inversely correlated with left ventricular wall thickness and end-diastolic interventricular septum thickness, left ventricular mass index, and HR.

Patients with LC have an increased resting HR due to hyperdynamic circulation, increased circulating catecholamines, and cirrhotic cardiomyopathy [48,49]. Kwon et al. [50] demonstrated that resting HR is associated with all-cause mortality in LT recipients and showed that patients with $\mathrm{HR}>80$ beats/min are significantly associated with a higher risk for all-cause mortality (hazard ratio 1.83) compared to patients with $\mathrm{HR} \leq 65$ beats/ min.

\section{Coronary Artery Disease and LT}

Plotkin et al. [51] reported an overall mortality of $50 \%$ over a follow-up period of 1-3 years involving 32 LT recipients with angiographically proven coronary artery disease (CAD). Al- 
though that study was performed 20 years ago, the importance of CAD should not be underestimated during LT surgery.

A previous study reported the prevalence of $\mathrm{CAD}$, which was detected by coronary computed tomography angiography in LT candidates [52]. Obstructive CAD ( $\geq 50 \%$ luminal narrowing) did not differ between cirrhotic and control groups $(7.2 \%$ vs. $7.9 \%, \mathrm{P}=0.646$ ); however, non-obstructive $\mathrm{CAD}$ was more prevalent in matched cirrhotic cases $(30.6 \%$ vs. $23.4 \%, \mathrm{P}=0.001)$. In that study, the risk factors of obstructive CAD were older age, male sex, hypertension, diabetes mellitus, and alcoholic cirrhosis. Similarly, Kazankov et al. [53] reported that the prevalence of CAD is not significantly different between cirrhotic patients and controls. However, they reported that cirrhotic patients have a markedly higher coronary calcium score than controls and have a higher prevalence of extensive ( $\geq 5$ coronary segments involved; $45 \%$ vs. $18 \%, \mathrm{P}=0.01)$ and multi-vessel coronary disease ( $\geq 2$ vessels involved; $75 \%$ vs. $53 \%, \mathrm{P}=0.02$ ).

Kong et al. [54] reported that a coronary calcium score $>400$ is an important predictor of early cardiovascular complications such as nonfatal myocardial infarction, serious arrhythmia, and cardiac death after LT and found that older age, male sex, and diabetes mellitus are associated with a coronary calcium score > 400 in LT recipients [55].

\section{Conclusions}

LT causes significant and heavy stress on the cardiovascular system of patients with LC and cirrhotic cardiomyopathy, who may experience massive bleeding and transfusion, inferior vena cava clamping, severe hypotension, and postreperfusion syndrome. Therefore, anesthesiologists should understand the pathophysiology of ongoing and postoperative forthcoming cardiovascular derangement in LT candidates and must identify risky patients preoperatively for successful LT.

\section{ORCID}

Hye-Mee Kwon, https://orcid.org/0000-0001-7788-9555

Gyu-Sam Hwang, https://orcid.org/0000-0002-3627-1107

\section{References}

1. Landesberg G, Beattie WS, Mosseri M, Jaffe AS, Alpert JS. Perioperative myocardial infarction. Circulation 2009; 119: $2936-44$.

2. Watt KD, Pedersen RA, Kremers WK, Heimbach JK, Charlton MR. Evolution of causes and risk factors for mortality post-liver transplant: results of the NIDDK long-term follow-up study. Am J Transplant 2010; 10: 1420-7.

3. Van Wagner LB, Lapin B, Levitsky J, Wilkins JT, Abecassis MM, Skaro AI, et al. High early cardiovascular mortality after liver transplantation. Liver Transpl 2014; 20: 1306-16.

4. Vernon G, Baranova A, Younossi ZM. Systematic review: the epidemiology and natural history of non-alcoholic fatty liver disease and nonalcoholic steatohepatitis in adults. Aliment Pharmacol Ther 2011; 34: 274-85.

5. Anstee QM, Targher G, Day CP. Progression of NAFLD to diabetes mellitus, cardiovascular disease or cirrhosis. Nat Rev Gastroenterol Hepatol 2013; 10: 330-44.

6. Wiese S, Hove JD, Bendtsen F, Møller S. Cirrhotic cardiomyopathy: pathogenesis and clinical relevance. Nat Rev Gastroenterol Hepatol 2014; 11: 177-86.

7. Ma Z, Lee SS. Cirrhotic cardiomyopathy: getting to the heart of the matter. Hepatology 1996; 24: 451-9.

8. Møller S, Henriksen JH. Cirrhotic cardiomyopathy: a pathophysiological review of circulatory dysfunction in liver disease. Heart 2002; 87: 9-15.

9. Møller S, Henriksen JH. Cardiovascular complications of cirrhosis. Gut 2008; 57: 268-78.

10. Møller S, Henriksen JH. Cardiovascular dysfunction in cirrhosis. Pathophysiological evidence of a cirrhotic cardiomyopathy. Scand J Gastroenterol 2001; 36: 785-94.

11. Bernardi M, Maggioli C, Dibra V, Zaccherini G. QT interval prolongation in liver cirrhosis: innocent bystander or serious threat? Expert Rev Gastroenterol Hepatol 2012; 6: 57-66.

12. Zaky A, Bendjelid K. Appraising cardiac dysfunction in liver transplantation: an ongoing challenge. Liver Int 2015; 35: 12-29.

13. Wong F, Liu P, Lilly L, Bomzon A, Blendis L. Role of cardiac structural and functional abnormalities in the pathogenesis of hyperdynamic circulation and renal sodium retention in cirrhosis. Clin Sci (Lond) 1999; 97: 259-67.

14. Ripoll C, Yotti R, Bermejo J, Bañares R. The heart in liver transplantation. J Hepatol 2011; 54: 810-22.

15. Harinstein ME, Flaherty JD, Ansari AH, Robin J, Davidson CJ, Rossi JS, et al. Predictive value of dobutamine stress echocardiography for coronary artery disease detection in liver transplant candidates. Am J Transplant 2008; 8: 1523-8.

16. Safadi A, Homsi M, Maskoun W, Lane KA, Singh I, Sawada SG, et al. Perioperative risk predictors of cardiac outcomes in patients undergoing liver transplantation surgery. Circulation 2009; 120: 1189-94.

17. Nicolau-Raducu R, Gitman M, Ganier D, Loss GE, Cohen AJ, Patel H, et al. Adverse cardiac events after orthotopic liver transplantation: a cross-sectional study in 389 consecutive patients. Liver Transpl 2015; 21: 13-21. 
18. Aydinalp A, Bal U, Atar I, Ertan C, Aktaş A, Yildirir A, et al. Value of stress myocardial perfusion scanning in diagnosis of severe coronary artery disease in liver transplantation candidates. Transplant Proc 2009; 41: 3757-60.

19. Davidson CJ, Gheorghiade M, Flaherty JD, Elliot MD, Reddy SP, Wang NC, et al. Predictive value of stress myocardial perfusion imaging in liver transplant candidates. Am J Cardiol 2002; 89: 359-60.

20. Day CP, James OF, Butler TJ, Campbell RW. QT prolongation and sudden cardiac death in patients with alcoholic liver disease. Lancet 1993; 341: 1423-8.

21. Bernardi M, Calandra S, Colantoni A, Trevisani F, Raimondo ML, Sica G, et al. Q-T interval prolongation in cirrhosis: prevalence, relationship with severity, and etiology of the disease and possible pathogenetic factors. Hepatology 1998; 27: 28-34.

22. Zierler K, Wu FS. Insulin acts on Na, K, and Ca currents. Trans Assoc Am Physicians 1988; 101: 320-5.

23. Zhong J, Hwang TC, Adams HR, Rubin LJ. Reduced L-type calcium current in ventricular myocytes from endotoxemic guinea pigs. Am J Physiol 1997; 273: H2312-24.

24. Shin WJ, Kim YK, Song JG, Kim SH, Choi SS, Song JH, et al. Alterations in QT interval in patients undergoing living donor liver transplantation. Transplant Proc 2011; 43: 170-3.

25. Lee HM, Park SK, Moon YJ, Kim JW, Kim SK, Sang BH, et al. Arrhythmogenic potential develops rapidly at graft reperfusion before the start of hypotension during living-donor liver transplantation. Korean J Anesthesiol 2016; 69: 37-43.

26. Berger RD, Kasper EK, Baughman KL, Marban E, Calkins H, Tomaselli GF. Beat-to-beat QT interval variability: novel evidence for repolarization lability in ischemic and nonischemic dilated cardiomyopathy. Circulation 1997; 96: 1557-65.

27. Huh IY, Park ES, Kim KI, Lee AR, Hwang GS. Alteration of the QT variability index in end-stage liver disease. Korean J Anesthesiol 2014; 66: 199-203.

28. Nazar A, Guevara M, Sitges M, Terra C, Solà E, Guigou C, et al. LEFT ventricular function assessed by echocardiography in cirrhosis: relationship to systemic hemodynamics and renal dysfunction. J Hepatol 2013; 58: 51-7.

29. Shin WJ, Song JG, Jun IG, Moon YJ, Kwon HM, Jung K, et al. Effect of ventriculo-arterial coupling on transplant outcomes in cirrhotics: analysis of pressure-volume curve relations. J Hepatol 2017; 66: 328-37.

30. Bushyhead D, Kirkpatrick JN, Goldberg D. Pretransplant echocardiographic parameters as markers of posttransplant outcomes in liver transplant recipients. Liver Transpl 2016; 22: 316-23.

31. Sampaio F, Pimenta J, Bettencourt N, Fontes-Carvalho R, Silva AP, Valente J, et al. Systolic and diastolic dysfunction in cirrhosis: a tissueDoppler and speckle tracking echocardiography study. Liver Int 2013; 33: 1158-65.

32. Jansen C, Cox A, Schueler R, Schneider M, Lehmann J, Praktiknjo M, et al. Increased myocardial contractility identifies decompensated cirrhotic patients requiring liver transplantation. Liver Transpl 2018; 24: 15-25.

33. Dowsley TF, Bayne DB, Langnas AN, Dumitru I, Windle JR, Porter TR, et al. Diastolic dysfunction in patients with end-stage liver disease is associated with development of heart failure early after liver transplantation. Transplantation 2012; 94: 646-51.

34. Starling MR. Left ventricular-arterial coupling relations in the normal human heart. Am Heart J 1993; 125: 1659-66.

35. Sagawa K, Suga H, Shoukas AA, Bakalar KM. End-systolic pressure/volume ratio: a new index of ventricular contractility. Am J Cardiol 1977; 40: 748-53.

36. Chen CH, Fetics B, Nevo E, Rochitte CE, Chiou KR, Ding PA, et al. Noninvasive single-beat determination of left ventricular end-systolic elastance in humans. J Am Coll Cardiol 2001; 38: 2028-34.

37. Kelly RP, Ting CT, Yang TM, Liu CP, Maughan WL, Chang MS, et al. Effective arterial elastance as index of arterial vascular load in humans. Circulation 1992; 86: 513-21.

38. Yotti R, Ripoll C, Benito Y, Catalina MV, Elízaga J, Rincón D, et al. Left ventricular systolic function is associated with sympathetic nervous activity and markers of inflammation in cirrhosis. Hepatology 2017; 65: 2019-30.

39. Jensen MT, Suadicani P, Hein HO, Gyntelberg F. Elevated resting heart rate, physical fitness and all-cause mortality: a 16-year follow-up in the Copenhagen Male Study. Heart 2013; 99: 882-7.

40. Greenland P, Daviglus ML, Dyer AR, Liu K, Huang CF, Goldberger JJ, et al. Resting heart rate is a risk factor for cardiovascular and noncardiovascular mortality: the Chicago Heart Association Detection Project in Industry. Am J Epidemiol 1999; 149: 853-62.

41. Fox K, Ford I, Steg PG, Tendera M, Robertson M, Ferrari R. Heart rate as a prognostic risk factor in patients with coronary artery disease and left-ventricular systolic dysfunction (BEAUTIFUL): a subgroup analysis of a randomised controlled trial. Lancet 2008; 372: 817-21.

42. Jensen MT, Marott JL, Allin KH, Nordestgaard BG, Jensen GB. Resting heart rate is associated with cardiovascular and all-cause mortality after adjusting for inflammatory markers: the Copenhagen City Heart Study. Eur J Prev Cardiol 2012; 19: 102-8.

43. Mani AR, Montagnese S, Jackson CD, Jenkins CW, Head IM, Stephens RC, et al. Decreased heart rate variability in patients with cirrhosis relates to the presence and degree of hepatic encephalopathy. Am J Physiol Gastrointest Liver Physiol 2009; 296: G330-8.

44. Møller S, Henriksen JH. Cirrhotic cardiomyopathy. J Hepatol 2010; 53: 179-90.

45. Kim YK, Lee K, Hwang GS, Cohen RJ. Sympathetic withdrawal is associated with hypotension after hepatic reperfusion. Clin Auton Res 2013; 23: 123-31.

46. Møller S, Iversen JS, Henriksen JH, Bendtsen F. Reduced baroreflex sensitivity in alcoholic cirrhosis: relations to hemodynamics and humoral systems. Am J Physiol Heart Circ Physiol 2007; 292: H2966-72.

47. Song JG, Kim YK, Shin WJ, Hwang GS. Changes in cardiovagal baroreflex sensitivity are related to increased ventricular mass in patients 
with liver cirrhosis. Circ J 2012; 76: 2807-13.

48. Trevisani F, Sica G, Mainquà P, Santese G, De Notariis S, Caraceni P, et al. Autonomic dysfunction and hyperdynamic circulation in cirrhosis with ascites. Hepatology 1999; 30: 1387-92.

49. Groszmann RJ. Hyperdynamic circulation of liver disease 40 years later: pathophysiology and clinical consequences. Hepatology 1994; 20: 1359-63.

50. Kwon HM, Jun IG, Jung KW, Moon YJ, Shin WJ, Song JG, et al. Pretransplant resting heart rate and its association with all-causemortality in liver transplant recipients. Transplant Proc 2017; 49: 1092-6.

51. Plotkin JS, Scott VL, Pinna A, Dobsch BP, De Wolf AM, Kang Y. Morbidity and mortality in patients with coronary artery disease undergoing orthotopic liver transplantation. Liver Transpl Surg 1996; 2: 426-30.

52. An J, Shim JH, Kim SO, Lee D, Kim KM, Lim YS, et al. Prevalence and prediction of coronary artery disease in patients with liver cirrhosis: a registry-based matched case-control study. Circulation 2014; 130: 1353-62.

53. Kazankov K, Munk K, Øvrehus KA, Jensen JM, Siggaard CB, Grønbaek H, et al. High burden of coronary atherosclerosis in patients with cirrhosis. Eur J Clin Invest 2017; 47: 565-73.

54. Kong YG, Kang JW, Kim YK, Seo H, Lim TH, Hwang S, et al. Preoperative coronary calcium score is predictive of early postoperative cardiovascular complications in liver transplant recipients. Br J Anaesth 2015; 114: 437-43.

55. Kong YG, Ha TY, Kang JW, Hwang S, Lee SG, Kim YK. Incidence and predictors of increased coronary calcium scores in liver transplant recipients. Transplant Proc 2015; 47: 1933-8. 